\title{
Структурно-спектроскопические исследования эпитаксиальных слоев GaAs, выращенных на податливых подложках на основе сверхструктурного слоя и протопористого кремния
}

\author{
(С) П.В. Середин ${ }^{1,2}$, Д.Л. Голощапов ${ }^{1}$, Ю.Ю. Худяков ${ }^{1}$, И.Н. Арсентьев ${ }^{3}$, Д.Н. Николаев ${ }^{3}$, \\ H.A. Пихтин ${ }^{3}$, C.O. Слипченко ${ }^{3}$, Harald Leiste ${ }^{4}$ \\ ${ }^{1}$ Воронежский государственный университет, \\ 394018 Воронеж, Россия \\ ${ }^{2}$ Уральский фредеральный университет им. первого Президента России Б.Н. Ельцина, \\ 620002 Екатеринбург, Россия \\ ${ }^{3}$ Физико-технический институт им. А.Ф. Иоффре Российской академии наук, \\ 194021 Санкт-Петербург, Россия \\ ${ }^{4}$ Karlsruhe Nano Micro Facility H.-von-Helmholtz-Platz 1 \\ 76344 Eggenstein-Leopoldshafen, Germany \\ E-mail: paul@phys.vsu.ru; arsentyev@mail.ioffe.ru \\ Поступила в Редакцию 3 сентября 2020 г. \\ В окончательной редакции 10 сентября 2020 г. \\ Принята к публикации 10 сентября 2020 г.
}

Цель работы заключалась в исследовании влияния нового типа податливых подложек на основе сверхструктурного слоя (SL) AlGaAs и слоя протопористого кремния (proto-Si), сформированного на $c$ - $\mathrm{Si}$, на практическую реализацию и особенности эпитаксиального роста слоя GaAs в методе MOCVD. Впервые показано, что низкотемпературный рост эпитаксиальных пленок GaAs высокого кристаллического качества может быть реализован за счет использования податливых подложек SL/proto-Si. Введение SL в состав податливой подложки в дополнение к proto-Si позволяет нивелировать ряд негативных эффектов низкотемпературного роста, снизить уровень напряжений в эпитаксиальном слое, защитить от автолегирования атомами кремния, сократить число технологических операций по росту переходных буферных слоев, улучшить структурные и морфологические характеристики эпитаксиального слоя.

Ключевые слова: $\mathrm{GaAs}, \mathrm{Si}$, por-Si, сверхструктурный слой.

DOI: $10.21883 /$ FTP.2021.01.50394.9518

\section{1. Введение}

Интеграция оптических функциональных элементов $\mathrm{A}^{\mathrm{III}} \mathrm{B}^{\mathrm{V}}$ и кремниевой схемы обработки электрических сигналов является ключом к созданию многофункциональных оптоэлектронных полупроводниковых приборов будущего.

Известны разнообразные попытки развить эту идеологию, однако, несмотря на наметившиеся успехи в интеграции $\mathrm{A}^{\mathrm{III}} \mathrm{B}^{\mathrm{V}}$ c Si и появление приборных применений, гетероэпитаксиальное выращивание полупроводников $\mathrm{A}^{\mathrm{III}} \mathrm{B}^{\mathrm{V}}$ на кремнии все еще остается сложной задачей. Внушительная разница между постоянными кристаллической решетки (4.1\%) и коэффициентами теплового расширения (120.4\%) GaAs и подложки $\mathrm{Si}$ при $300 \mathrm{~K}$ ведут к появлению большого числа точечных дефектов и дислокаций, что весьма серьезно затрудняет рост пленки хорошего качества. Разница в коэффициентах термического расширения также способствует образованию большого количества дислокаций и трещин в эпитаксиальном слое в процессе его охлаждения.

Хорошо известно, что дислокации необходимы для перераспределения механической энергии и деформации несоответствия кристаллических решеток. Однако при этом они весьма часто отрицательно влияют на оптические и электрические свойства активных слоев, резко сокращают срок работы оптоэлектронных устройств [1]. Управление дислокациями все еще является важной задачей.

Помимо возникновения протяженных дефектов при эпитаксиальном росте структур $\mathrm{A}^{\mathrm{III}} \mathrm{B}^{\mathrm{V}} / \mathrm{Si}$ часто либо происходит разделение фаз, возникают антифазные домены [2], двойники, дефекты упаковки, наблюдается островковый рост, либо имеет место небольшая флуктуация состава, что приводит к появлению структурных дефектов, действующих как центры безызлучательной рекомбинации $[3,4]$. Образование островков, происходящее во время эпитаксиального роста $\mathrm{A}^{\mathrm{III}} \mathrm{B}^{\mathrm{V}} / \mathrm{Si}$, также крайне нежелательно [5], поскольку оно создает центры нуклеации для дефектов.

Рассматривая реализованные технологические подходы к эпитаксиальному выращиванию структур $\mathrm{A}^{\mathrm{III}} \mathrm{B}^{\mathrm{V}} / \mathrm{Si}$, следует отметить, что для фильтрации дислокаций, прорастающих от гетероинтерфейса в активную область приборной гетероструктуры, используют многопериодные сверхрешетки, слои с чередующейся двумерной (2D) и трехмерной (3D) морфологией, слои с плавным изменением состава и т.д., а также их комбинации. Такие буферные слои представляют собой сложные, многослойные гетероструктуры, толщины которых могут достигать нескольких десятков микрометров. Хотя 
использование буферных слоев позволяет эффективно снижать плотность прорастающих дислокаций в активной зоне гетероструктуры, их плотность на гетероинтерфейсе $\mathrm{A}^{\mathrm{III}} \mathrm{B}^{\mathrm{V}} / \mathrm{Si}$ остается высокой, ухудшая финальные характеристики конечной структуры.

Не менее интересным подходом при выращивании интегрированных структур является использование переходных слоев $\mathrm{Si}_{1-x} \mathrm{Ge}_{x} / \mathrm{Ge} / \mathrm{Si}$ [6,7]. Однако использование такой технологии в большой степени оправдано лишь в частном случае создания кремний-германиевой электроники, для которой разница параметров решетки в гетеропаре $\mathrm{Ge} / \mathrm{Si}$ минимальна, что весьма упрощает эпитаксиальный рост. При этом на основе ряда эффектов, например, эффекта Киркендалла, для соединений $\mathrm{Si}_{1-x} \mathrm{Ge}_{x}$ в последние годы открываются новые возможности для приложений в оптоэлектронике [8].

В ряде наших предыдущих работ [9-12] мы показали, что для создания гибридных гетероструктур $\mathrm{GaAs} / \mathrm{Si}$ c высокими функциональными характеристиками весьма перспективно применение податливой подложки, которая представляет собой переходный нанопористый слой кремния (por-Si), созданный непосредственно на монокристалле $c$-Si методом электрохимического травления.

Еще одним технологическим приемом для создания нового типа податливых подложек может стать введение в ее состав сверхструктурных слоев (SL) $\mathrm{A}^{\mathrm{III}} \mathrm{B}^{\mathrm{V}}$. Известно, что сверхструктуры не только обладают уникальными свойствами, отличными от свойств неупорядоченных твердых растворов того же состава, но и способны подстроиться под любую (в частности „идеальную“) длину связи и любой угол связи, тем самым минимизировать высокую степень рассогласования кристаллических решеток [13-16]. В уже выполненных нами работах [16-19] в системах полупроводниковых соединений $\mathrm{A}^{\mathrm{III}} \mathrm{B}^{\mathrm{V}}(\mathrm{AlGaAs}, \mathrm{GaInP}, \mathrm{InGaAs}$ и др.) нам удалось показать перспективность данного подхода.

Обзор литературы показывает, что информации об одновременном применении двух описанных методик для создания податливой подложки SL/proto-Si и последующего роста на ней эпитаксиального слоя $\mathrm{A}^{\mathrm{III}} \mathrm{B}^{\mathrm{V}}$ нет.

Поэтому целью данной работы является структурноспектроскопическое исследование влияния нового вида податливых подложек, созданных на основе сверхструктурного слоя $\mathrm{AlGaAs}$ и слоя протопористого кремния proto-Si, на практическую реализацию и особенности эпитаксиального роста при использовании метода MOCVD (metalloorganic chemical vapor deposition) слоев $\mathrm{GaAs}$, а также сопоставление полученных результатов с технологией роста на стандартных подложках

\section{2. Материалы и методы}

Исследуемые структуры выращивались методом MOC-гидридной эпитаксии (газофазной эпитаксии из металлоорганических соединений, MOCVD) на установке EMCORE GS3100 с реактором вертикального типа и резистивным нагревом подложкодержателя. Давление в реакторе - 77 Торр, скорость вращения подложкодержателя - 1000 об/мин. В качестве источников служили триметилгаллий $\mathrm{Ga}\left(\mathrm{CH}_{3}\right)_{3}$, триметилалюминий $\mathrm{Al}\left(\mathrm{CH}_{3}\right)_{3}$ и арсин $\mathrm{AsH}_{3}$. В качестве газа-носителя использовался водород $\left(\mathrm{H}_{2}\right)$. Для роста структур были использованы подложки $\mathrm{Si}(100)$ с разориентацией $3^{\circ}$ к (110).

Рост структур происходил по следующей технологии.

На первом этапе, аналогично тому, как это было сделано в наших предыдущих работах $[10,11]$, на поверхности $\mathrm{Si}$ был сформирован протопористый слой путем травления исходной подложки в течение 1 мин в растворе следующего состава: 1 часть $\mathrm{HF}+1$ часть уксусной кислоты +40 частей азотной кислоты.

Далее обработанная подложка $\mathrm{Si}$ отжигалась в $\mathrm{AsH}_{3}$ в течение 20 мин при температуре $750^{\circ} \mathrm{C}$. После этого температура понижалась в течение 7 мин до $450^{\circ} \mathrm{C}$ при потоке $\mathrm{AsH}_{3}$.

На следующем этапе были выращены эпитаксиальный слой AlAs толщиной 10 нм и GaAs толщиной $30 \mathrm{HM}$, после чего температура повышалась в течение 7 мин до $550^{\circ} \mathrm{C}$ при потоке $\mathrm{AsH}_{3}$. При этой температуре для структуры A выращивался слой GaAs толщиной 360 нм, а для структуры В был выращен сверхструктурный слой (SL) AlGaAs толщиной $\sim 100$ нм и сверху слой GaAs толщиной 700 нм.

Рентгенофазовый анализ образцов проводили методом рентгеновской дифракции с использованием дифрактометра ДРОН 7 с медным анодом. Исследование кристаллического качества образцов было выполнено с применением высокоразрешающей рентгеновской дифракции с использованием картирования обратного $q$-пространства образцов. Исследования проводили методом рентгеновской дифракции (XRD) на дифрактометре Seifert 3003 HR с 4-кружным гониометром и монохроматизированным излучением меди с длиной волны $\mathrm{Cu} K_{\alpha 1}=1.5405 \AA$.

Микроскопические исследования качества гетероинтерфейсов были выполнены на электронном микроскопе Libra 120 Carl Zeiss.

Изучение морфологии поверхности проводили с использованием сканирующего зондового микроскопа Femtoscan-001 NT MDT в режиме атомно-силовой микроскопии.

\section{3. Экспериментальные данные}

\section{1. Рентгеновская дифракция}

Фазовый анализ и исследование кристаллического качества эпитаксиальных слоев GaAs, как выше сказано, были выполнены нами с применением методов рентгеновской дифракции.

На рис. 1 приведены обзорные дифрактограммы, полученные в геометрии Брегга-Брентано. Хорошо видно, что на дифрактограммах присутствуют лишь разрешенные для данной геометрии и правил отбора 


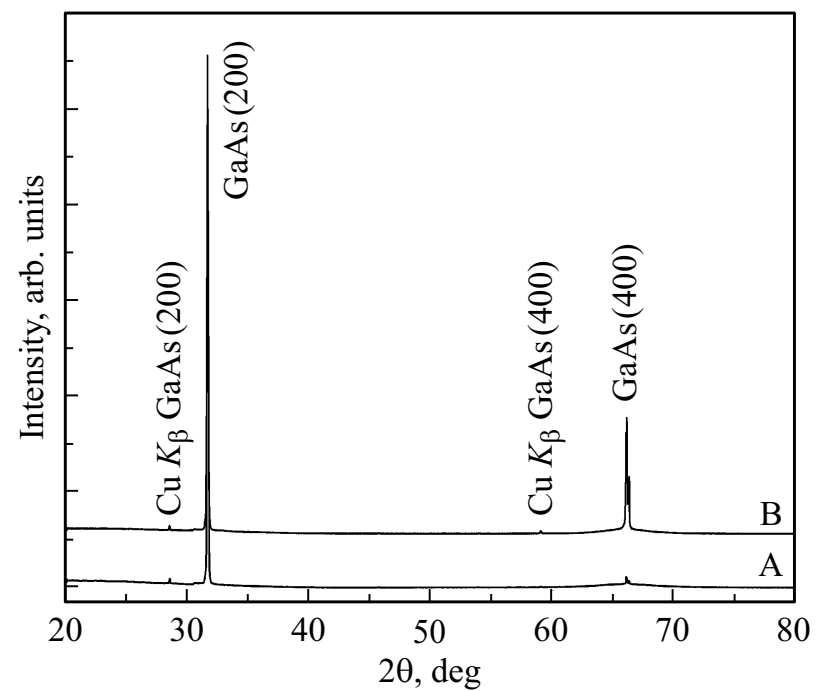

Рис. 1. Обзорные дифрактограммы гетероструктур, выращенных на податливых подложках proto-Si(100) и $\mathrm{SL} /$ proto-Si(100).

четные рефлексы (200) и (400), что указывает на факт роста эпитаксиальной пленки в монокристаллическом состоянии. Однако, как следует из экспериментальных данных, рефлекс (400) на дифрактограмме образца В, выращенного на податливой подложке SL/proto-Si, имеет значительно большую интенсивность, чем в случае гетероструктуры А. Это может являться следствием как более высокого кристаллического качества эпитаксиального слоя GaAs в гетероструктуре В, так и близости его ориентации к (100).

Расчет параметров кристаллической решетки эпитаксиальных слоев в плоскости и в направлении роста, а также деформации и релаксации компонент гетероструктуры был выполнен на основе данных высокоразрешающей дифракции рентгеновских лучей (HRXRD). От всех образцов были собраны две карты обратного пространства (RSM) вокруг симметричного (004) и асимметричного (511) отражений. Карты приведены на рис. 2. Вертикальная ось на картах соответствует компоненте вектора обратной решетки $q_{z}$, перпендикулярной плоскости роста эпитаксиальной пленки GaAs, т.е. параллельно направлению [001], а горизонтальная ось $q_{x}$ соответствует компоненте вектора в плоскости, параллельной поверхности вдоль направления [110].

Анализ результатов показывает, что на симметричной (рис. 2,a) и асимметричной (рис. 2,c) картах обратного пространства гетероструктуры А, выращенной на подложке proto-Si, присутствуют рефлексы (400) эпитаксиального слоя GaAs и подложки. В то же время для гетероструктуры $\mathrm{B}$, выращенной на подложке $\mathrm{SL} /$ proto-Si, симметричная карта вокруг узла (400) coдержит лишь отражения от $\mathrm{GaAs}$ и $\mathrm{Si}$ (рис. 2,b), а на карте асимметричного рассеяния (рис. $2, d$ ) дополнительно присутствует рефлекс (511) от SL. Отсутствие узла от SL на карте (400) может быть обусловлено низкой интенсивностью этого рефлекса по сравнению с отражениями (400) от $\mathrm{Si}$ и GaAs.
Параметры кристаллической решетки в направлении роста $\left(a^{\perp}\right)$ и в плоскости роста $\left(a^{\|}\right)$для всех слоев гетероструктур А и В могут быть определены исходя из данных, полученных при анализе карт обратного q-пространства для симметричного (400) и асимметричного (511) рефлексов. Так, параметр $a^{\perp}$ может быть определен на основе информации от симметричного отражения (400) и

$$
\frac{h^{2}}{a^{\perp 2}}=q_{400}^{\perp 2}+q_{400}^{\| 2}
$$

или асимметричного рефлекса и

$$
\frac{h^{2}}{a^{\perp 2}}=q_{500}^{\perp 2},
$$

в то время как $a^{\|}$задается следующим соотношением с учетом данных лишь асимметричного отражения (511):

$$
\frac{k^{2}+l^{2}}{a^{\| 2}}=q_{011}^{\| 2} \text {. }
$$

Здесь $q$ с индексами - координаты узлов $(h k l)$ в обратном $q$-пространстве.

В соответствии с линейной теорией упругости, вводя коэффициент Пуассона $v$ для кристалла с кубической симметрией, параметр кристаллической решетки эпитаксиальной пленки GaAs c учетом внутренних напряжений, обозначаемый стандартно как $a^{v}$, можно рассчитать следующим образом:

$$
a_{\mathrm{GaAs}}^{v}=a_{\mathrm{GaAs}}^{\perp} \frac{1-v_{\mathrm{GaAs}}}{1+v_{\mathrm{GaAs}}}+a_{\mathrm{GaAs}}^{\|} \frac{2 v_{\mathrm{GaAs}}}{1+v_{\mathrm{GaAs}}} .
$$

Аналогично выражение (4) может быть записано и для параметра SL:

$$
a_{\mathrm{SL}}^{v}=a_{\mathrm{SL}}^{\perp} \frac{1-v_{\mathrm{SL}}}{1+v_{\mathrm{SL}}}+a_{\mathrm{SL}}^{\|} \frac{2 v_{\mathrm{SL}}}{1+v_{\mathrm{SL}}} .
$$

B расчетах для GaAs мы использовали коэффициент Пуассона $v_{\mathrm{GaAs}}=0.310$ [20], а для сверхслоя данный коэффициент положили равным его значению для твердого pаствора $\mathrm{Al}_{0.50} \mathrm{Ga}_{0.50} \mathrm{As}$, т. е. $v_{\mathrm{SL}}=v_{\mathrm{AlGaAs}}=0.3045$.

Рассчитанные компоненты параметра решетки для эпитаксиального слоя GaAs, SL и подложки $\mathrm{Si}$, а также параметры решетки GaAs и SL с учетом внутренних напряжений представлены в таблице. Кроме того в таблице представлены значения параметров решетки для слоя GaAs гомоэпитаксиальной структуры C, изученной в нашей предыдущей работе [10].

Следует отметить, что параметр кристаллической решетки SL, рассчитанный из данных HRXRD с учетом внутренних напряжений, по своей величине весьма сильно отличается от параметра кристаллической решетки твердого раствора AlGaAs аналогичного состава. На карте (511) асимметричного отражения мы отметили положение рефлекса от $\mathrm{AlGaAs}$. 
Результаты высокоразрешающей рентгеновской дифракции

\begin{tabular}{|c|c|c|c|c|c|c|}
\hline Образец & Соединение & $\begin{array}{c}\text { Параметр решетки, } \\
\AA\end{array}$ & $\begin{array}{c}\text { Параметр решетки } \\
\text { с учетом напряжений } \\
a^{v}, \AA\end{array}$ & $\begin{array}{c}\text { Остаточные } \\
\text { напряжения, } \\
\%\end{array}$ & $\begin{array}{c}\text { Коэффициент } \\
\text { релаксации }\end{array}$ & $\begin{array}{c}\text { Дисторсия, } \\
D\end{array}$ \\
\hline \multirow[b]{2}{*}{ A } & $\mathrm{Si}$ & 5.4315 & 5.4315 & - & - & - \\
\hline & GaAs & $\begin{array}{l}a_{\mathrm{GaAs}}^{\perp}=5.6512 \\
a_{\mathrm{GaAs}}^{\|}=5.6592\end{array}$ & 5.6549 & 0.03 & 1.009 & 0.965 \\
\hline \multirow{4}{*}{ B } & $\mathrm{Si}$ & 5.4315 & 5.4315 & - & - & - \\
\hline & SL & $\begin{array}{l}a_{\mathrm{SL}}^{\perp}=5.5141 \\
a_{\mathrm{SL}}^{\|}=5.6534\end{array}$ & 5.5791 & - & - & - \\
\hline & GaAs & $\begin{array}{l}a_{\mathrm{GaAs}}^{\perp}=5.6524 \\
a_{\mathrm{GaAs}}^{\|}=5.6534\end{array}$ & 5.6528 & -0.008 & 0.998 & 0.995 \\
\hline & GaAs & $a_{\mathrm{GaAs}}^{\perp}=a_{\mathrm{GaAs}}^{\|}=5.6532[10]$ & 5.6532 & - & - & - \\
\hline
\end{tabular}

Значительное уменьшение параметра кристаллической решетки SL связано с атомным упорядочением в ней, что характерно для трехкомпонентных полупроводниковых составов III-V с $x \approx 0.50$ [13]. Однако необходимо отметить, что определенный нами в предыдущих работах [21] параметр решетки сверхструктурной фазы упорядочения $a_{\mathrm{AlGaAs}_{2}}$ отличается от параметра $a_{\mathrm{SL}}^{v}$, ровно как и от теоретически рассчитанного для сверхструктуры [22].

На наш взгляд, уменьшение параметра решетки $a_{\mathrm{SL}}^{v}$ от тех значений, которые мы наблюдали ранее для сверхструктуры AlGaAs, может быть связано с особенностями низкотемпературного роста, реализованного в этой работе.

С использованием полученных данных о компонентах параметра кристаллической решетки и теории упругости для напряженного слоя GaAs компоненты деформации (в плоскости и в направлении роста эпитаксиального слоя) могут быть определены следующим образом:

$$
\begin{gathered}
\varepsilon^{\|}=\frac{\Delta a^{\|}}{a_{\mathrm{Si}}}=\frac{a_{f}^{\|}-a_{\mathrm{Si}}}{a_{\mathrm{Si}}}, \\
\varepsilon^{\perp}=\frac{\Delta a^{\perp}}{a_{\mathrm{Si}}}=\frac{a_{f}^{\perp}-a_{\mathrm{Si}}}{a_{\mathrm{Si}}} .
\end{gathered}
$$

Здесь $a_{f}^{\|}, a_{f}^{\perp}-$ параметры кристаллической решетки в плоскости и направлении роста эпитаксиальной пленки, $a_{\mathrm{Si}}$ - параметр решетки монокристаллической подложки $\mathrm{Si}, a_{\mathrm{Si}}=5.431 \AA[20]$.

На основе данных высокоразрешающей дифрактометрии можем рассчитать величину остаточных напряжений в эпитаксиальной пленке GaAs $[5,23]$ и коэффициент релаксации [24,25] соответственно как

$$
\begin{gathered}
\frac{a_{\mathrm{GaAs}}^{v}-a_{\mathrm{GaAs}}}{a_{\mathrm{GaAs}}}, \\
\frac{a_{\mathrm{GaAs}}^{v}-a_{\mathrm{Si}}}{a_{\mathrm{GaAs}}-a_{\mathrm{Si}}},
\end{gathered}
$$

где $a_{\mathrm{GaAs}}$ - параметр ненапряженного (bulk) GaAs.
Хорошо известно, что деформация в плоскости роста связана линейно с деформацией в направлении роста через соотношение:

$$
D=\frac{\varepsilon^{\perp}}{\varepsilon^{\|}},
$$

где $D-$ коэффициент дисторсии (искажения) кристаллической решетки эпитаксиального слоя GaAs.

Рассчитанные с учетом (7)-(9) значения коэффициента релаксации, остаточных напряжений и величины дисторсии $D$ в пленке GaAs представлены в таблице.

Дополнительную информацию о структуре эпитаксиальных пленок может дать анализ положения и формы узлов на картах обратного пространства образцов. Типичная форма узла в обратном пространстве для релаксированного эпитаксиального слоя с дислокациями есть эллипс [26]. Появление дефектов (дислокаций) в эпитаксиальном слое, релаксация эпитаксиального слоя, а также остаточные напряжения в нем отражаются на картах обратного пространства. Уширение узла обратной решетки на картах может происходить в двух направлениях - параллельно вектору дифракции, $q^{\|}$, и перпендикулярно ему, $q^{\perp}[26,27]$. Для оценки ширины узлов (HWHM) GaAs, подложки Si, а также SL на карте асимметричного рассеяния (511) были построены их сечения в плоскости и в направлении роста. Сечения приведены на рис. 3.

Следует отметить, что узлы от монокристаллической подложки кремния $\mathrm{Si}(100)$ на картах симметричного и асимметричного рассеяния (см. рис. 2) имеют наклон к плоскости дифракции, в то время как рефлексы от эпитаксиальных слоев GaAs и SL такого наклона практически не имеют. Это является следствием того факта, что для роста была использована подложка $\mathrm{Si}(100)$, имеющая разориентаци $3^{\circ}$ к направлению (110).

Уширение узла образной решетки GaAs перпендикулярно вектору дифракции для гетероструктуры А по отношению к гетероструктуре В (см. рис. 3, $a$ ) 

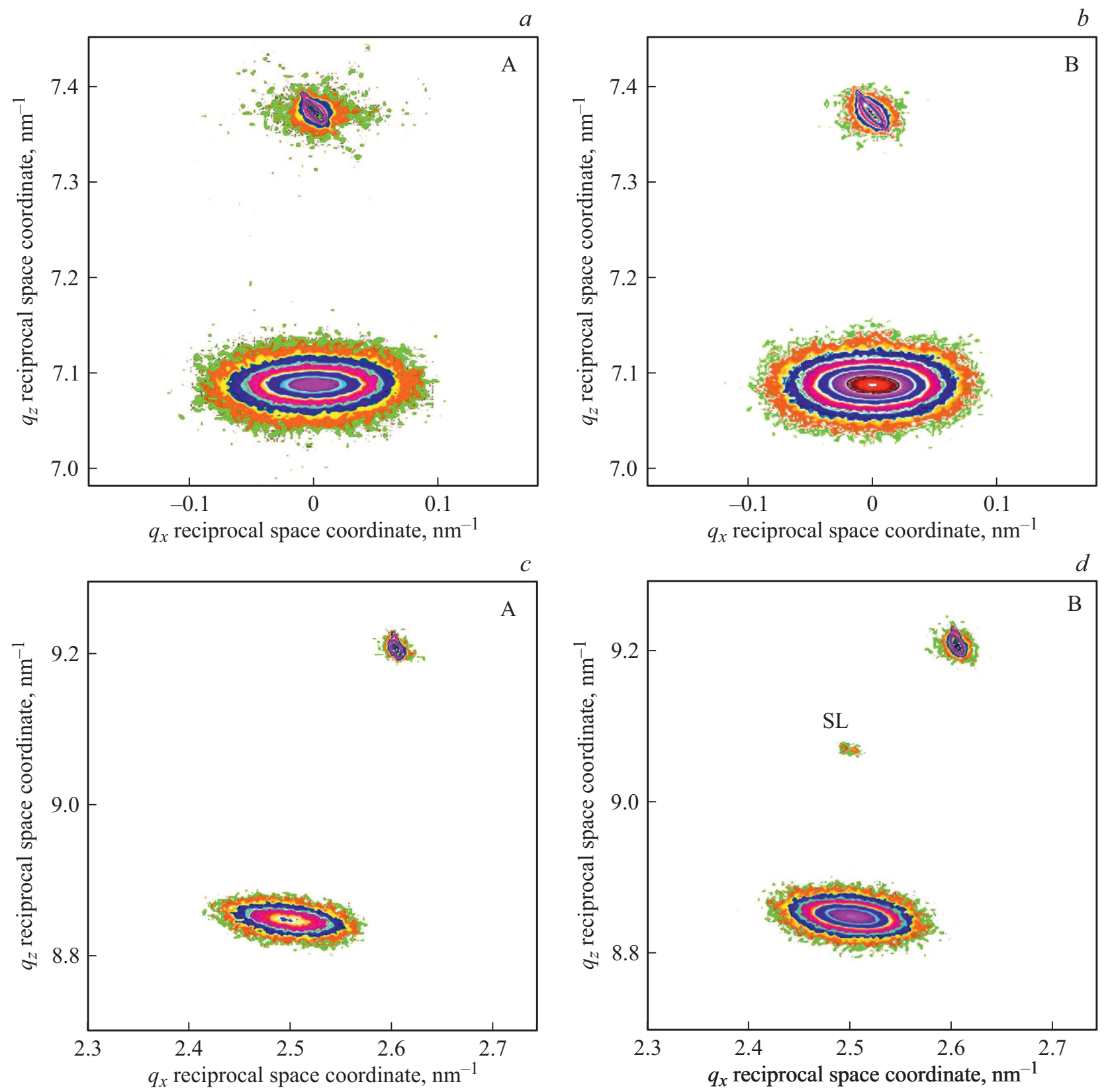

Рис. 2. Карты дифрагированного излучения в обратном q-пространстве, собранные около симметричного отражения (004) $(a, b)$ и асимметричного отражения (511) $(c, d)$ для гетероструктур GaAs/proto-Si(100) (образец А) и GaAs/SL/proto-Si(100) (образец B).

является следствием большей мозаичности эпитаксиального слоя, вызванной дислокациями несоответствия, способствующими ослаблению упругой энергии деформации кристаллической решетки. Из этого следует, что введение слоя SL в состав податливой подложки снижает мозаичность пленки GaAs по сравнению с использованием податливой подложки proto-Si. Как хорошо видно из полученных данных, использование SL снизило ширину узла GaAs на величину $\sim 25 \%$.

Сопоставление полуширины сечений узлов в направлении вектора рассеяния в обратном пространстве для эпитаксиальных слоев $\mathrm{GaAs}$ (рис. 4,b) и подложки $\mathrm{Si}$ (рис. $3, d$ ) позволяет сделать заключение о том, что вдоль направления дифракции деформация (strain) имеет значительные изменения по глубине эпитаксиального слоя GaAs. Однако при росте GaAs на податливой подложке SL/proto-Si величина деформации в слое существенно меньше (снижение до 20\%), чем при его формировании на подложке proto-Si.

Анализируя форму сечения узла (511) обратной решетки для SL (рис. 3,e,f), можно заметить, что она является асимметричной и имеет несколько максимумов. Такое разделение максимумов интенсивности, вероятнее всего, напрямую связано с релаксацией деформации в слое SL в направлении дифракции [28]. 

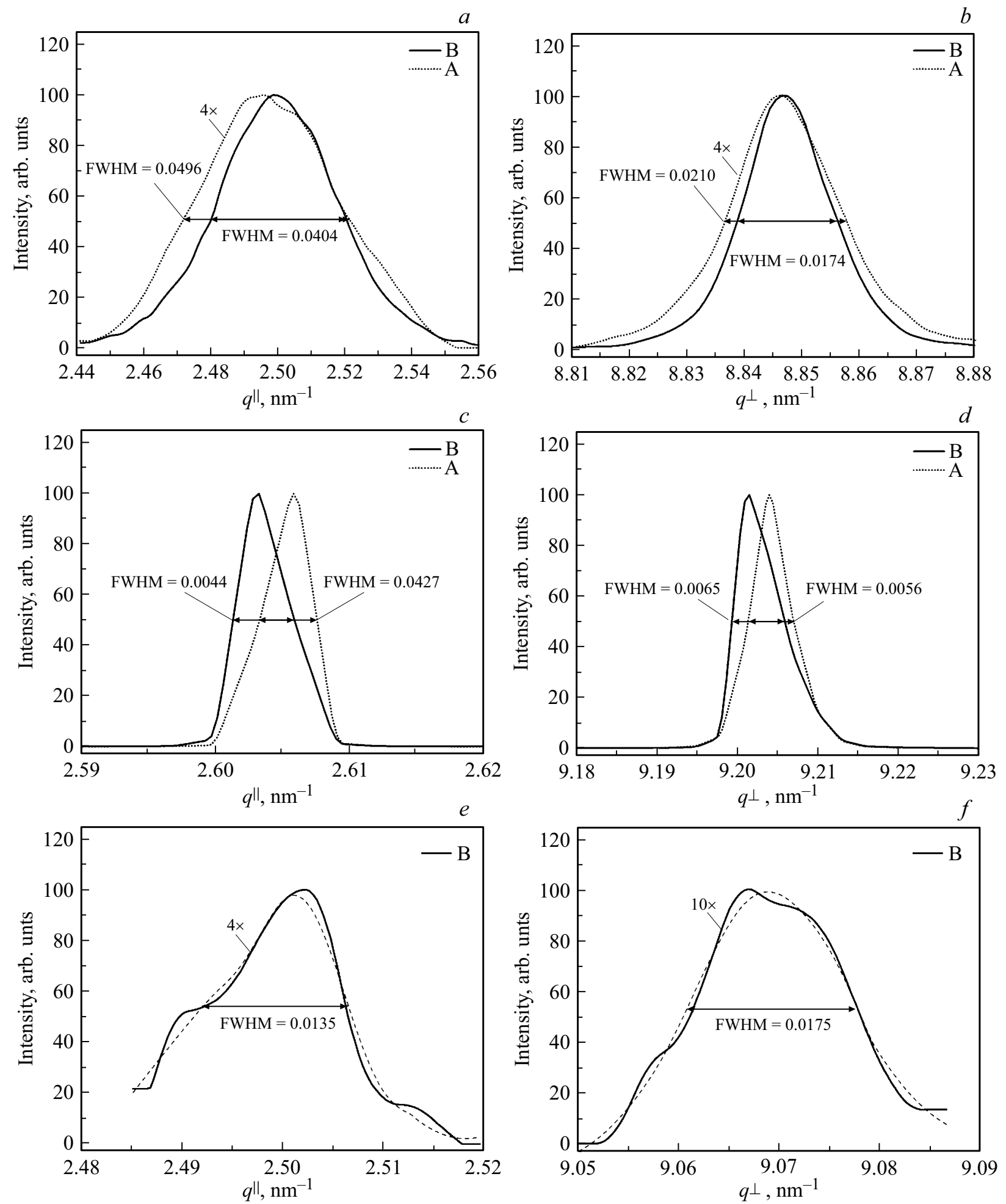

Рис. 3. Сечения узлов $(511) \mathrm{GaAs}(a, b), \mathrm{Si}(c, d)$ и $\mathrm{SL}(e, f)$ в плоскости и в направлении роста для гетероструктур на податливых подложках proto-Si $(100)$ и $\mathrm{SL} /$ proto-Si $(100)$.

\section{2. Атомно-силовая и сканирующая электронная микроскопия}

Исследования морфологии эпитаксиальных слоев $\mathrm{GaAs}$, а также их сколов для определения качества гете- роинтерфейсов были выполнены нами с использованием методов сканирующей электронной и атомно-силовой микроскопии.

На рис. 4 приведены результаты электронной микроскопии, которые подтверждают для обоих образ- 

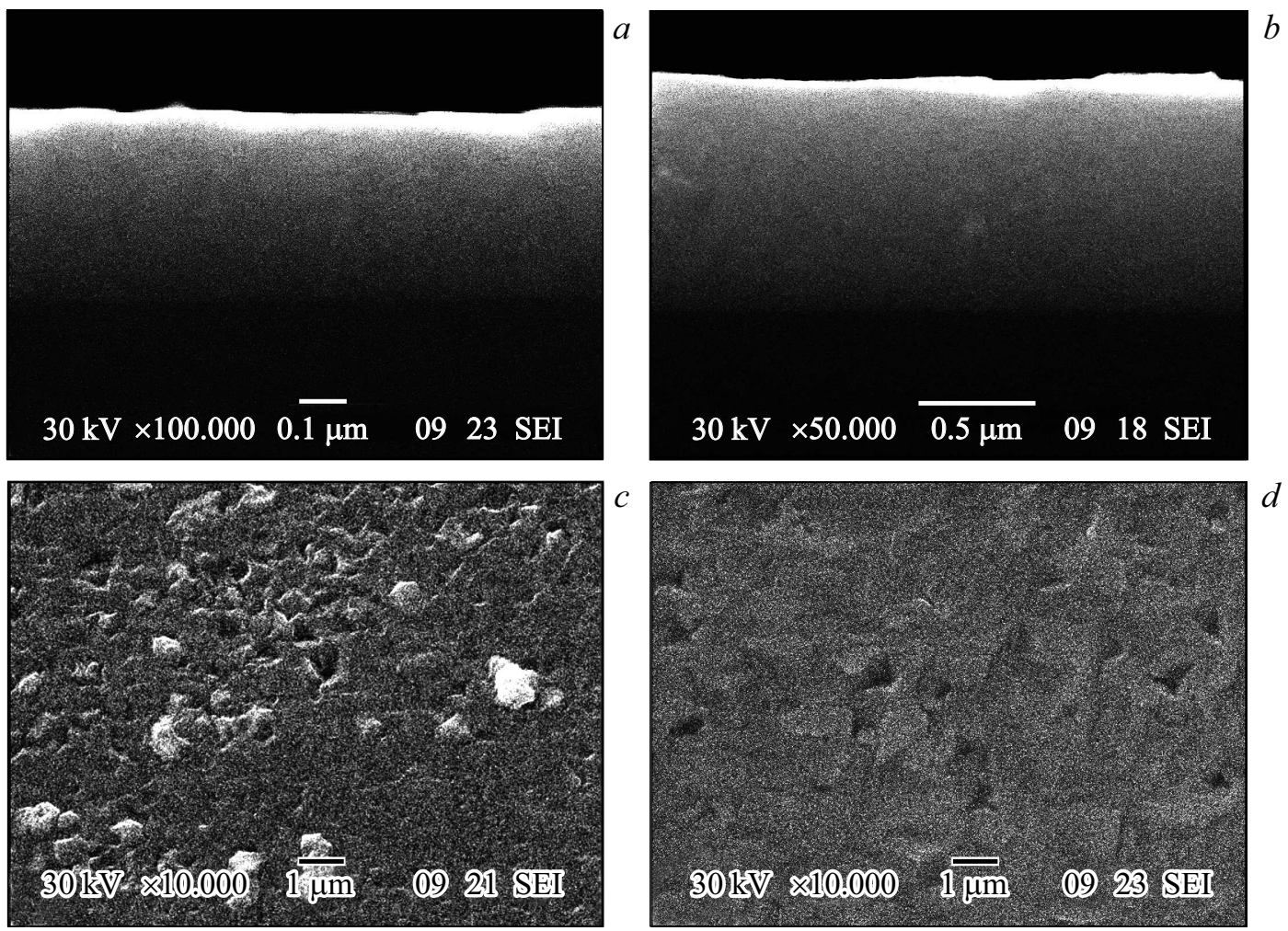

Рис. 4. Результаты электронной микроскопии с различной степенью увеличения для образцов интегрированных структур: (a,c) $\mathrm{GaAs} /$ proto-Si(100) (образец A); $(b, d)-\mathrm{GaAs} / \mathrm{SL} /$ proto-Si(100) (образец B).

цов заданные технологически толщины пленки GaAs (рис. 4, $a, b)$. Что же касается качества поверхности эпитаксиальных слоев GaAs, выращенных на двух типах податливых подложек, то на поверхности гетероструктур, как это следует из экспериментальных данных (рис. 4, $c, d$ ), присутствуют дефекты различной формы в виде углублений, а также шарообразных включений, которые в соответствии с данными работы [29] могут быть кластерами мышьяка. Следует отметить, что на поверхности эпитаксиального слоя, полученного на подложке SL/proto-Si, по сравнению с образцом, полученным на подложке proto-Si, наблюдается значительно меньшее число дефектов в виде углублений, а также практически отсутствуют шарообразные включения. Известно, что накопленная деформация в эпитаксиальном слое высвобождается за счет появления шероховатости поверхности и трехмерных островков с увеличением времени роста [30].

Более детально микрорельеф поверхности был изучен с использованием атомно-силовой микроскопии. Результаты исследования представлены на рис. 5. Хорошо видно, что поверхность эпитаксиального слоя обоих образцов образована плотной упаковкой блоков (мозаики). При этом для гетероструктуры, полученной на proto-Si, размеры блоков GaAs значительно меньше, чем при росте на податливой подложке SL/proto-Si (рис. 5,c,d). Кроме того, в последнем случае блоки имеют более правильную форму прямоугольников. Исследования в фазовом контрасте показывают, что на поверхности присутствует фактически одна фаза, однако для гетероструктуры А отмечается наличие на поверхности мелких кластеров $(\sim 50 \times 50 \mathrm{Hм})$, которые практически отсутствуют на поверхности гетероструктуры В. Этот результат совпадает с данными электронной микроскопии.

\section{4. Обсуждение полученных результатов и выводы}

Наши предыдущие исследования показали, что рост эпитаксиальных пленок полупроводниковых соединений группы GaAs и нитридов $\mathrm{A}^{\mathrm{III}} \mathrm{N}$ с использованием податливых подложек, содержащих слой нанопористого или протопористого кремния, имеет ряд преимуществ по сравнению с ростом на монокристаллическом кремнии $c$-Si.

Результаты текущей работы еще раз подтверждают, что рост эпитаксиальных слоев GaAs высокого кристаллического качества на кремнии может быть реализован за счет использования подложек $\mathrm{Si}$ с малой степенью разориентации и слоя протопористого кремния, сформированного на $c$-Si.

Предварительная обработка кремниевой подложки с формированием протопористого слоя позволила вырастить на ней методом MOCVD эпитаксиальную пленку $\mathrm{GaAs}$ в монокристаллическом состоянии с хорошими 

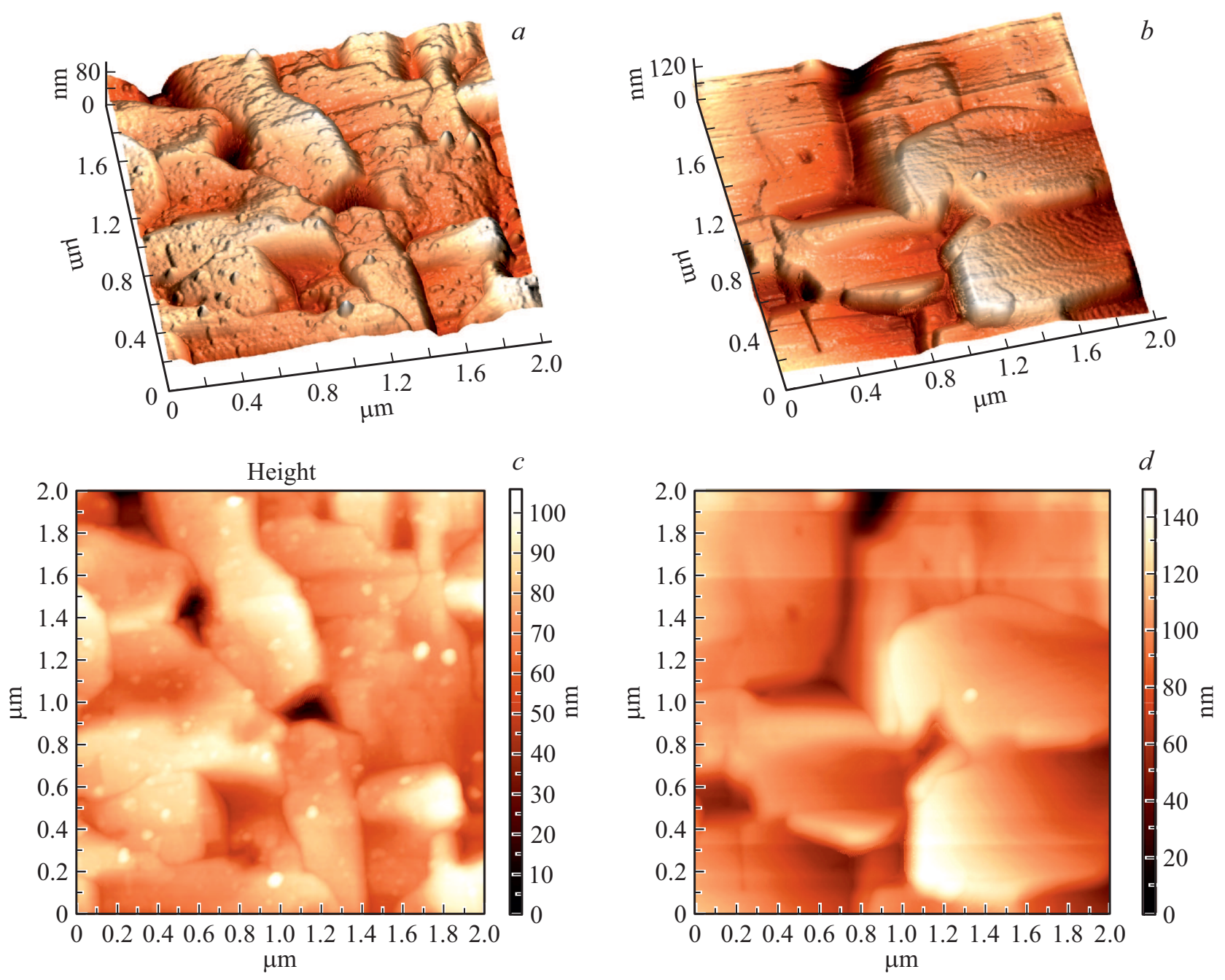

Рис. 5. Результаты исследования морфологии поверхности эпитаксиального слоя GaAs, выращенного на подложках proto- $\mathrm{Si}(100(a, c)$ и $\mathrm{SL} /$ proto- $\mathrm{Si}(100)(b, d)$. Участок поверхности $2 \times 2$ мкм, 3D-режим $(a, b)$ и 2D-режим $(c, d)$.

структурными и оптическими характеристиками, без использования высокой степени разориентации подложки $\left(>6^{\circ}\right)$, что подтверждено данными структурно-спектроскопических методов анализа [10,11].

Полученные в нашей текущей работе данные для гетероструктуры А, выращенной на податливой подложке proto-Si с отклонением от сингулярного направления $3^{\circ}$ к (110), находятся в хорошем согласии с результатами наших предыдущих исследований $[10,11]$. Однако эпитаксиальный слой GaAs в гетероструктуре А был выращен при значительно более низкой температуре $\left(T=550^{\circ} \mathrm{C}\right)$, чем образец с аналогичной структурой в $[10,11]\left(T \approx 700^{\circ} \mathrm{C}\right)$, что в свою очередь положительно отразилось на микроструктурном качестве пленки GaAs. По данным сканирующей электронной микроскопии, в эпитаксиальном слое образца А практически не наблюдается объемных дефектов, в то время как при росте в более высокотемпературном режиме в эпитаксиальной пленке $\mathrm{GaAs}$, выращенной на подложке $c$-Si(100) c разориентацией подложки $\sim 3^{\circ}$ к [110], были хорошо видны объемные дефекты $[10,11]$.
Хотя поверхность эпитаксиального слоя GaAs, выросшего на податливых подложках двух типов, имеет блочную структуру, при росте на податливой подложке SL/proto-Si блоки не только имеют более правильную форму прямоугольников, но они и значительно крупнее, чем при использовании подложки proto-Si. Более того, в соответствии с данными высокоразрешающей рентгеновской дифракции RSM и рамановской спектроскопии уровень напряжений в эпитаксиальном слое GaAs гетероструктуры, выращенной на податливой подложке $\mathrm{SL} /$ proto-Si, практически на 50\% меньше, чем для гетероструктуры, сформированной на подложке proto-Si. Введение SL позволяет эффективно перераспределять напряжения, возникающие из-за рассогласования параметров кристаллических решеток, подстраиваясь под длину связи в гетеропаре, и минимизировать это рассогласование [13-16].

В нашем предыдущем исследовании [21] мы показали, что параметр сверхструктурной фазы $\mathrm{AlGaAs}$ c учетом внутренних напряжений имел величину 
$a_{\mathrm{AlGaAs}}^{v}=5.64 \AA$, в то время как определенный в текущей работе $a_{\mathrm{SL}}^{v}=5.58 \AA$.

Уменьшение параметра может быть связано с тем фактом, что при низкотемпературном росте в решетку SL может встраиваться углерод по механизмам низкотемпературного автолегирования при MOCVD-росте [31].

До наших исследований в работе [1] был продемонстрирован успех технологического подхода по использованию сверхрешеток $\mathrm{AlAs} / \mathrm{GaAs}$ для выращивания слоя $\mathrm{GaAs}$ при пониженной температуре $\left(620^{\circ} \mathrm{C}\right)$ с низкой плотностью антифазных границ, без трещин методом молекулярно-лучевой эпитаксии на кремниевых подложках (001), на которых был предварительно сформирован суспендированный буфер Ge. Необходимо отметить, что такой подход позволил успешно подавлять дислокации, негативные явления, возникающие за счет термической деформации, а также устранять антифазные границы с использованием точно ориентированной подложки. Однако, чтобы добиться удовлетворительного оптического качества гетероструктур на основе описанного подхода, были выращены весьма толстые переходные слои ( 10 мкм) при температурах, превышающих использованные нами в данной работе. Более того, в этой работе авторы не приводят прямых структурных данных (полученных методом рентгеновской дифракции), чтобы достоверно оценить структурное качество выращенных образцов. Наши экспериментальные данные и их сравнение с известными литературными источниками показывают, что введение SL в состав податливой подложки позволяет нивелировать ряд негативных эффектов низкотемпературного роста, перераспределить возникающие напряжения в кристаллической решетке, а также сократить число технологических операций при росте переходных буферных слоев.

Полученные данные послужат важным материалом для понимания основ физики и технологии интегрированных гетероструктур $\mathrm{A}^{\mathrm{III}} \mathrm{B}^{\mathrm{V}} / \mathrm{Si}$, способствуя развитию их потенциала для применения в устройствах оптоэлектроники.

\section{Благодарности}

We acknowledge the Karlsruhe Nano Micro Facility (KNMF, www.kit.edu/knmf) of the Forschungszentrum Karlsruhe for provision of access to instruments at their laboratories.

\section{Финансирование}

Работа выполнена при финансовой поддержке гранта Российского научного фонда 19-72-10007.

Свою часть работы П.В. Середин выполнил при поддержке гранта Министерства науки и высшего образования Российской Федерации № FZGU-2020-0036 в рамках государственного задания вузам.

Технологические исследования эпитаксиальных процессов методом MOCVD были выполнены по государственной плановой программе ФТИ им. А.Ф. Иоффе.

\section{Конфликт интересов}

Авторы заявляют, что у них нет конфликта интересов.

\section{Список литературы}

[1] A. Ballabio, S. Bietti, A. Scaccabarozzi, L. Esposito, S. Vichi, A. Fedorov, A. Vinattieri, C. Mannucci, F. Biccari, A. Nemcsis, L. Toth, L. Miglio, M. Gurioli, G. Isella, S. Sanguinetti. Sci. Rep., 9, (2019). doi: 10.1038/s41598-019-53949-x

[2] A.G. Taboada, T. Kreiliger, C.V. Falub, F. Isa, M. Salvalaglio, L. Wewior, D. Fuster, M. Richter, E. Uccelli, P. Niedermann, A. Neels, F. Mancarella, B. Alén, L. Miglio, A. Dommann, G. Isella, H. von Känel. Appl. Phys. Lett., 104, 022112 (2014). doi: $10.1063 / 1.4861864$

[3] Q. Li, K.W. Ng, K.M. Lau. Appl. Phys. Lett., 106, 072105 (2015). doi: 10.1063/1.4913432

[4] I. Prieto, R. Kozak, O. Skibitzki, M.D. Rossell, T. Schroeder, R. Erni, H. von Känel. Small, 13, 1603122 (2017). doi: $10.1002 /$ smll.201603122

[5] A. Ohtake, T. Mano, Y. Sakuma. Sci. Rep., 10, (2020). doi: 10.1038/s41598-020-61527-9

[6] Encyclopedia of Nanoscience and Nanotechnology, ed. by H.S. Nalwa (American Scientific Publishers, Stevenson Ranch, Calif, 2004).

[7] F. Schäffler. Semicond. Sci. Technol., 12, 1515 (1997). doi: 10.1088/0268-1242/12/12/001

[8] V. Sivadasan, S. Rhead, D. Leadley, M. Myronov. Semicond. Sci. Technol., 33, 024002 (2018). doi: 10.1088/1361-6641/aaa329

[9] P.V. Seredin, D.L. Goloshchapov, A.S. Lenshin, A.M. Mizerov, D.S. Zolotukhin. Phys. E: Low-Dim. Syst. Nanostructures, 104, 101 (2018). doi: 10.1016/j.physe.2018.07.024

[10] P.V. Seredin, A.S. Lenshin, D.S. Zolotukhin, I.N. Arsentyev, A.V. Zhabotinskiy, D.N. Nikolaev. Phys. E: Low-Dim. Syst. Nanostructures, 97, 218 (2018). doi: 10.1016/j.physe.2017.11.018

[11] P.V. Seredin, A.S. Lenshin, D.S. Zolotukhin, I.N. Arsentyev, D.N. Nikolaev, A.V. Zhabotinskiy. Phys. B: Condens. Matter, 530, 30 (2018). doi: 10.1016/j.physb.2017.11.028

[12] P.V. Seredin, A.S. Lenshin, A.M. Mizerov, H. Leiste, M. Rinke. Appl. Surf. Sci., 476, 1049 (2019). doi: 10.1016/j.apsusc.2019.01.239

[13] A. Zunger. MRS Bull., 22, 20 (1997). doi: $10.1557 / \mathrm{S} 0883769400033364$

[14] P.V. Seredin, A.V. Glotov, E.P. Domashevskaya, I.N. Arsentyev, D.A. Vinokurov, I.S. Tarasov. Phys. B: Condens. Matter, 405, 4607 (2010). doi: 10.1016/j.physb.2010.07.026

[15] P.V. Seredin, A.S. Lenshin, V.M. Kashkarov, A.N. Lukin, I.N. Arsentiev, A.D. Bondarev, I.S. Tarasov. Mater. Sci. Semicond. Process., 39, 551 (2015). doi: 10.1016/j.mssp.2015.05.067

[16] P.V. Seredin, A.V. Glotov, E.P. Domashevskaya, I.N. Arsentyev, D.A. Vinokurov, I.S. Tarasov. Appl. Surf. Sci., 267, 181 (2013). doi: 10.1016/j.apsusc.2012.09.053

[17] E.P. Domashevskaya, P.V. Seredin, A.N. Lukin, L.A. Bityutskaya, M.V. Grechkina, I.N. Arsentyev, D.A. Vinokurov, I.S. Tarasov. Surf. Interface Anal., 38, 828 (2006). doi: $10.1002 /$ sia.2306 
[18] P.V. Seredin, D.L. Goloshchapov, Yu.Yu. Khudyakov, A.S. Lenshin, A.N. Lukin, I.N. Arsentyev, T. Prutskij. Phys. B: Condens. Matter, 509, 1 (2017). doi: 10.1016/j.physb.2016.12.030

[19] T. Prutskij, P. Seredin, G. Attolini. J. Luminesc., 195, 334 (2018). doi: 10.1016/j.jlumin.2017.11.016

[20] Properties of Semiconductor Alloys: Group-IV, III-V and II-VI Semiconductors, 1st edn, ed. by Sadao Adachi (Wiley, Chichester, UK, 2009).

[21] P.V. Seredin, P. Domashevskaya, I.N. Arsentyev, D.A. Vinokurov, A.L. Stankevich, T. Prutskij. Semiconductors, 47, 1 (2013). doi: 10.1134/S106378261301020X

[22] S. Laref, S. Meçabih, B. Abbar, B. Bouhafs, A. Laref. Phys. B: Condens. Matter, 396, 169 (2007). doi: 10.1016/j.physb.2007.03.033

[23] C.S. Wong, N.S. Bennett, B. Galiana, P. Tejedor, M. Benedicto, J.M. Molina-Aldareguia, P.J. Mc Nally. Semicond. Sci. Technol., 27, 115012 (2012). doi: $10.1088 / 0268-1242 / 27 / 11 / 115012$

[24] D.K. Bowen, B.K. Tanner. High Resolution X-Ray Diffractometry and Topography (Taylor \& Francis, London-Bristol, PA, 1998).

[25] H. Fitouri, M.M. Habchi, A. Rebey. In: X-Ray Scatt., ed. by A.E. Ares (InTech, 2017) doi: 10.5772/65404

[26] V. Lashkaryov. Semicond. Phys. Quant. Electron. Optoelectron., 16, 265 (2013). doi: 10.15407/spqeo16.03.265

[27] Characterization of Semiconductor Heterostructures and Nanostructures (Elsevier, 2008).

[28] V. Bellani, C. Bocchi, T. Ciabattoni, S. Franchi, P. Frigeri, P. Galinetto, M. Geddo, F. Germini, G. Guizzetti, L. Nasi, M. Patrini, L. Seravalli, G. Trevisi. Eur. Phys. J. B, 56, 217 (2007). doi: 10.1140/epjb/e2007-00105-8

[29] P.S. Dobal, H.D. Bist, S.K. Mehta, R.K. Jain. Semicond. Sci. Technol., 11, 315 (1996). doi: 10.1088/0268-1242/11/3/008

[30] G. Zhao, H. Li, L. Wang, Y. Meng, Z. Ji, F. Li, H. Wei, S. Yang, Z. Wang. Sci. Rep., 7, (2017). doi: 10.1038/s41598-017-04854-8

[31] P.V. Seredin, A.V. Glotov, E.P. Domashevskaya, I.N. Arsentyev, D.A. Vinokurov, I.S. Tarasov. Phys. B: Condens. Matter, 405, 2694 (2010). doi: 10.1016/j.physb.2010.03.049

Редактор Л.В. Шаронова

\section{Structural-spectroscopic studies of epitaxial GaAs layers grown on supportive substrates based on a superstructure layer and protoporous silicon}

\author{
P.V. Seredin ${ }^{1,2}$, D.L. Goloshchapov ${ }^{1}$, \\ Yu.Yu. Khudyakov' , I.N. Arsentiev ${ }^{3}$, D.N. Nikolaev ${ }^{3}$, \\ N.A. Pikhtin ${ }^{3}$, S.O. Slipchenko ${ }^{3}$, Harald Leiste ${ }^{4}$ \\ ${ }^{1}$ Voronezh State University,
394018 Voronezh, Russia \\ 2 Ural Federal University, \\ 620002 Yekaterinburg, Russia \\ ${ }^{3}$ loffe Institute, \\ 194021 St. Petersburg, Russia \\ ${ }^{4}$ Karlsruhe Nano Micro Facility \\ H.-von-Helmholtz-Platz 1, \\ 76344 Eggenstein-Leopoldshafen, Germany
}

\begin{abstract}
The purpose of the work was to study the influence of compliant substrates of the new type on the basis of superstructure layer (SL) of $\mathrm{AlGaAs}$ and the layer of protoporous silicon (proto-Si) formed on $c-\mathrm{Si}$ on the practical obtaining and the features of the epitaxial GaAs growth. For the first time it was shown that low-temperature MOCVD growth of structurally perfect epitaxial GaAs films can be realized due to the use of compliant SL/proto-Si substrates. Introducing of SL into construction of the compliant substrate in addition to proto-Si makes it possible to level a number of the negative effects of low-temperature growth, to reduce stresses in the epitaxial layer, to prevent autodoping with silicon atoms, to diminish the number of technological operations concerning the growth of transient buffer layers and to improve structural and morphological characteristics of the epitaxial layer.
\end{abstract}

